\title{
Molecular weight of gastric mucus glycoprotein is a determinant of the degree of subsequent aspirin induced chronic gastric ulceration in the rat
}

\author{
P F BAGSHAW, D J MUNSTER, AND J G WILSON \\ From the University Department of Surgery Christchurch Clinical School of Medicine, Christchurch Hospital, \\ Christchurch, New Zealand
}

SUMmaRY Mucus was sampled from the gastric mucosal surface of anaesthetised rats. Three weeks later these rats were orally dosed each day with aspirin $(375 \mathrm{mg} / \mathrm{kg})$ for six months. Then the number and size of the aspirin induced chronic gastric ulcers were assessed. Gel filtration chromatography of the mucus samples showed that mucus glycoprotein was present in both high and low molecular weight forms. There was a natural variation between individual rats in the percentage of glycoprotein in the high molecular weight form (mean $=58 \cdot 9 \% ; \mathrm{SD}=9 \cdot 6 \% ; \mathrm{n}=23)$. This variation correlated strongly with the degree of subsequent aspirin induced chronic gastric ulceration $(r=-0 \cdot 85, p<0 \cdot 001)$. This is the first time that a pre-existent variability in a mucosal defence factor has been shown to predict susceptibility of the stomach to chronic ulceration.

It has been previously noted with some animal models of chronic gastric ulceration, that a wide range of responses, between individual animals, is obtained with a constant dose per unit weight of an ulcerogenic agent. Hence, Pfeiffer and MacPherson postulated that this might be because of the variability in some aspect of mucosal defence.'

The surface mucus layer is one of the gastric mucosal defence factors, and it has been shown to be different in the presence of gastric ulcer in man. ${ }^{2}$ Gastrectomy specimens from patients with this condition had a mucus layer which contained a smaller percentage of high molecular weight mucus glycoprotein, than similar specimens removed from patients with non-ulcer disease. It is not known, however, if this difference in the gastric mucus predates or follows the ulceration. We wished to investigate which might be the case, but as there was no obvious way to do this in man, an animal model of chronic gastric ulceration was used. Of those available, the chronic aspirin dosed rat $^{3}$ was chosen for study.

The aims of the present study were: (1) to develop a method for sampling rat gastric epithelial mucus that permitted recovery of the animal for subsequent

Address for correspondence: P F Bagshaw FRCS. University Department of Surgery, Christchurch Clinical School of Medicine, Christchurch Hospital. Private Bag. Christchurch. New Zealand.

Received for publication 7 July 1986. chronic dosing with aspirin; (2) to determine if the rat, like man, has high molecular weight and low molecular weight gastric mucus glycoprotein, and to measure the variability in the proportions of the two forms; (3) to establish whether or not there is a correlation between the gastric mucus glycoprotein molecular weight distribution and the susceptibility of the stomach to subsequent aspirin induced chronic ulceration.

\section{Methods}

IN VIVO COLLECTION OF ADHERENT SURFACE

GASTRIC MUCUS

Adult Sprague Dawley rats $(200-300 \mathrm{~g})$ were used throughout the study. Nonfasted animals were anaesthetised with halothane, and the abdomen and stomach were opened. The food cast and other intragastric particles were removed from the mucosa with forceps. Bile reflux was avoided by temporary occlusion of the pylorus with a non-crushing microvascular clamp. The edges of the opened stomach were sutured back around the periphery of the abdominal wound, using fine silk sutures, so that the mucosa presented a flat surface.

The end of a plastic autopipette tip was cut off obliquely and any sharp edges were sanded down. The tip was fitted onto a $10 \mathrm{ml}$ syringe and $1 \mathrm{ml}$ 
$1 \mathrm{mmol} / \mathrm{IEDTA}$ at $\mathrm{pH} 7 \cdot 4$ was drawn up. The opened end of the tip was drawn lightly across a portion of the mucosal surface, while applying slight suction on the syringe. The small quantity of mucus appearing in the EDTA solution in the syringe was ejected into a glass tube on ice. This procedure was repeated on another portion of the corpus and antrum, until the whole of the glandular mucosal surface was aspirated into $10 \mathrm{ml}$ of the EDTA solution. The sample was then stored at $-20^{\circ} \mathrm{C}$ until analysis.

The gastric and abdominal incisions were closed with fine Dexon sutures. The rat was then allowed to recover for three weeks before aspirin dosing began.

ANALYSIS OF GASTRIC MUCUS

After lyophilisation, the following were added to each mucus sample, to give a final volume of $5.5 \mathrm{ml}$ : $2.76 \mathrm{ml}$ water, $1.65 \mathrm{~g}$ cesium chloride (Analar grade, BDH Chemicals Ltd, Poole, England), and $3 \cdot 15 \mathrm{~g}$ guanidine $\mathrm{HCl}$ (Grade 1, Sigma Chemical Co, St Louis, USA). The guanidine was required to dissolve a large water insoluble mucus plug, which was invariably present. The mixture was homogenised for 20 seconds (Ultra-Turrax, TP 1ON tip, Janke \& Kunkel GMBH \& $\mathrm{Co} \mathrm{KG}$ ) and incubated at $37^{\circ} \mathrm{C}$ for 30 minutes. Undissolved fur and feed particles were removed by filtration through a $2.5 \mathrm{~cm}$ diameter circle of Whatman no 1 paper. Five millilitres of each sample was then centrifuged at $25^{\circ} \mathrm{C}$ in a Beckman SW50.1 swing-out rotor for 48 hours at $37000 \mathrm{rpm}$ (91000-164000 $\mathrm{xg}$ ). The resulting density gradient was fractionated with an Isco model 184 tube piercer (Lincoln, Nebraska, USA) into six $0.85 \mathrm{ml}$ fractions.

Density gradient and subsequent gel filtration fractions were assayed by the periodic acid Schiff (PAS) carbohydrate method of Mantle and Allen. Protein was measured by the method of Lowry et al, after precipitation with methanol. ${ }^{\circ}$

Some crude mucus samples were reduced for 65 hours at $37^{\circ} \mathrm{C}$ with $0.5 \mathrm{~mol} / \mathrm{l}$ mercaptoethanol in 0.5 $\mathrm{mol} / \mathrm{l}$ Tris at $\mathrm{pH} 8 \cdot 4$. These were dialysed against distilled water for 48 hours before lyophilisation and analysis.

Gel filtration of the density gradient separated mucus glycoprotein was performed on a $1.5 \mathrm{~cm}$ diameter column containing Sepharose $2 \mathrm{BCL}$ with a bed volume of $125 \mathrm{ml}$. Samples were eluted with $0 \cdot 2$ $\mathrm{mol} / \mathrm{l} \mathrm{NaCl}, 1 \mathrm{mmol} / 1$ EDTA, $0.02 \%$ w/v NaN 3 , pH 7.4 , into $2.9 \mathrm{ml}$ fractions. The percentage of the glycoprotein present in the high molecular weight form was calculated according to the following formula:

$$
\% \mathrm{HMW}=\frac{\sum_{40 \mathrm{ml}}^{60 \mathrm{ml}}\left(\mathrm{A}_{555}\right)}{\sum_{41 \mathrm{ml}}^{100 \mathrm{ml}}\left(\mathrm{A}_{555}\right)} \cdot 100 \%
$$

where $\mathrm{A}_{555}$ is the PAS carbohydrate assay absorbance (minus blank) of each fraction.

The sugar and amino acid composition of pooled gel filtration fractions were determined by the method of Zanetta et al. ${ }^{7}$ and by ion exchange chromatography (Dionex D300, Sunnyvale, California), respectively.

ASPIRIN DOSING AND ASSESSMENT OF MUCOSAL. DAMAGE

After recovery from mucus sampling, the rats were orally dosed, ${ }^{3}$ five days per week, in the morning, with a suspension of aspirin in approximately $1 \mathrm{ml} 1 \%$ methyl cellulose. Another group of rats that had not undergone mucus sampling was similarly dosed. The rats were weighed weekly, and dosages calculated, so that each one received $375 \mathrm{mg} / \mathrm{kg} /$ day of aspirin. Some animals were dosed with $1 \%$ methyl cellulose only; half of these had been mucus sampled. All animals were allowed water ad libitum, but were fasted overnight before, and for three hours after, each dosing episode. At other times they had free access to feed pellets (Snowflake, New Zealand).

Dosing was continued for six months and then the rats were killed, 48 hours after the final dose. Their stomachs were removed, pinned out on cork boards, and fixed in $4 \%$ formaldehyde in phosphate buffered normal saline.

Chronic ulcers were first identified by viewing the stomachs through a binocular operating microscope, at $\times 12$ magnification, under surface illumination. Using this stereoscopic assessment method, a lesion was considered to be a chronic ulcer if it met all of the following criteria: it had a clearly defined margin; substantial depth relative to the thickness of the mucosa; a rolled edge from surrounding mucosal oedema; white fibrous slough in its base. By comparison, an erosion appeared as a small, shallow lesion, surrounded by pale, flat mucosa.

In order to have some assessment of the area of ulceration the maximum diameter of each chronic ulcer was measured. A scoring system was used so that larger lesions were given proportionately higher scores (Table 1). For each rat, an 'ulcer score' was calculated by adding the separate 'scores' for each lesion present in its stomach.

Table 1 Ulcerscores

\begin{tabular}{lll}
\hline $\begin{array}{l}\text { Ulcersize } \\
(\mathrm{mm})\end{array}$ & Lesionscore & $\begin{array}{l}\text { Ulcers found }^{*} \\
(\mathrm{n})\end{array}$ \\
\hline$\leqslant 1$ & 1 & 42 \\
$1 \cdot 1-2 \cdot 0$ & 2 & 17 \\
$2 \cdot 1-3 \cdot 0$ & 3 & 10 \\
$>3$ & 4 & 10 \\
\hline${ }^{*}$ In 33 rats. & &
\end{tabular}


The specimens were coded so that the identity of the individual rat stomachs was unknown at the time of assessment. For each rat, the sites, number, and sizes of all chronic ulcers, were recorded. This stereoscopic assessment was repeated on 19 of the aspirin dosed rats by three additional observers, each of whom was blinded to the assessments of the others.

A histological assessment was subsequently made of each stomach. Thin paraffin sections were made at $10 \mu \mathrm{m}$ intervals through lesions identified as chronic ulcers by any of the four observers. Sections were also taken from lesions which were not considered to be chronic ulcers - for example, erosions, artefacts of tissue fixation, etc. These were stained with haematoxylin and eosin $(\mathrm{H} \& \mathrm{E})$ and $\mathrm{PAS} / \mathrm{Alcian}$ Blue (PAS/AB), and were examined microscopically. A lesion was considered to be a chronic ulcer if it met all of the following histological criteria: the lesion extended through the muscularis mucosae; there was local endarteritis obliterans; there was evidence of chronic inflammation and fibrosis in its base.

STATISTICAL ANALYSIS

Quoted correlations were determined by the Pearson correlation coefficient ( $r$ ), although equally significant values were obtained, in all cases, with the
Spearman test. The Mann-Whitney test was used for statistical comparisons of means.

\section{Results}

COLLECTION OF GASTRIC MUCUS

For this study mucus was aspirated from 23 rats. All survived the procedure and went on to subsequent dosing regimens.

Examination of sections of gastric mucosa, taken immediately after mucus sampling by the aspiration method, showed that many of the heavily PAS/AB stained surface epithelial cells were lost (Fig. 1a, b). There was, however, no damage to the deeper layers of the mucosa. Three weeks after the surgery, a complete layer of apparently normal deeply staining epithelial cells had reformed and, except near the sutures, the mucosal tissues appeared histologically normal (Fig. 1c).

\section{VARIABILITY IN THE MUCUS}

Forty six per cent of the carbohydrate and $2 \cdot 5 \%$ of the protein from the aspirated mucus samples appeared in the two densest ultracentrifuge fractions (Fig. 2). Similar results were obtained from mucus scraped from excised stomachs, using the method of Spee-Brand et al."
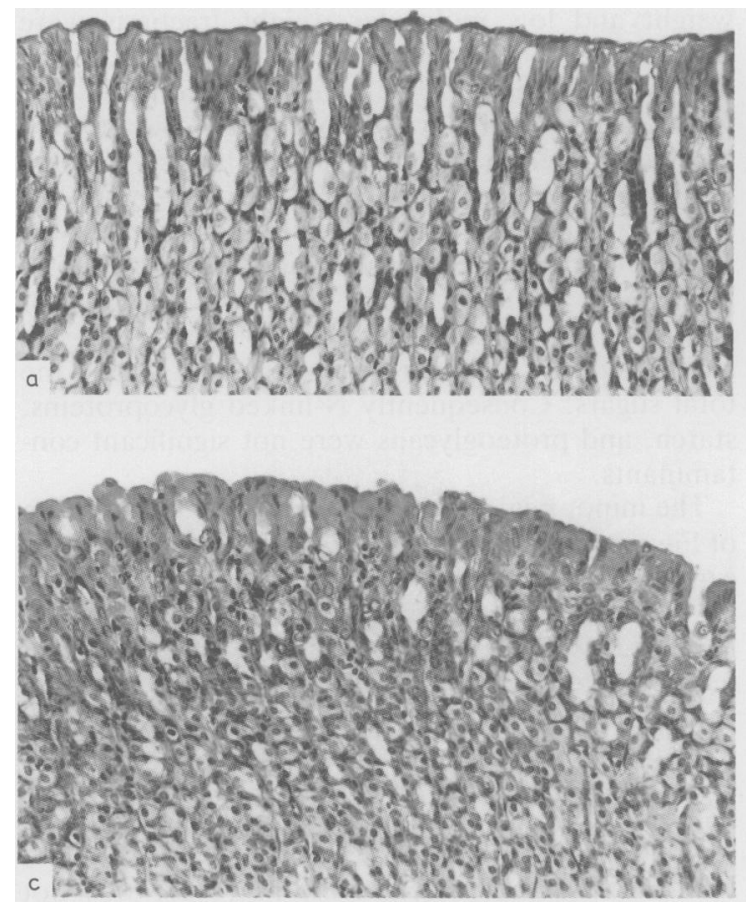

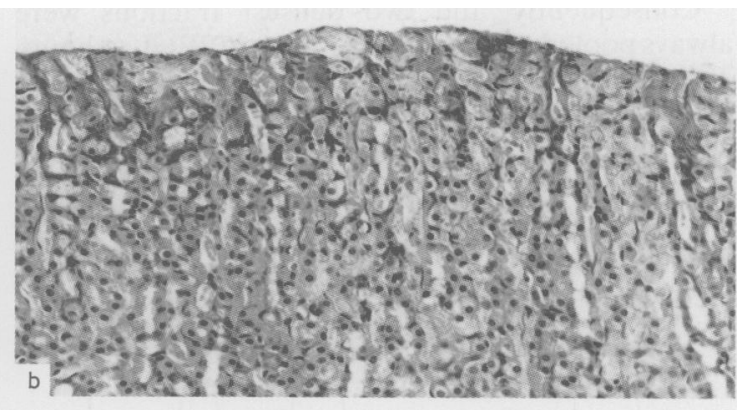

Fig. 1 Transverse sections of rat gastric mucosa. (All $H \& E$ and PAS/AB, $\times 70$ original magnification: (a) normal, showing heavily stained surface epithelial cells containing mucus glycoprotein; (b) immediately after mucus aspiration; (c) three weeks after aspiration, showing renewed surface epithelial layer. 


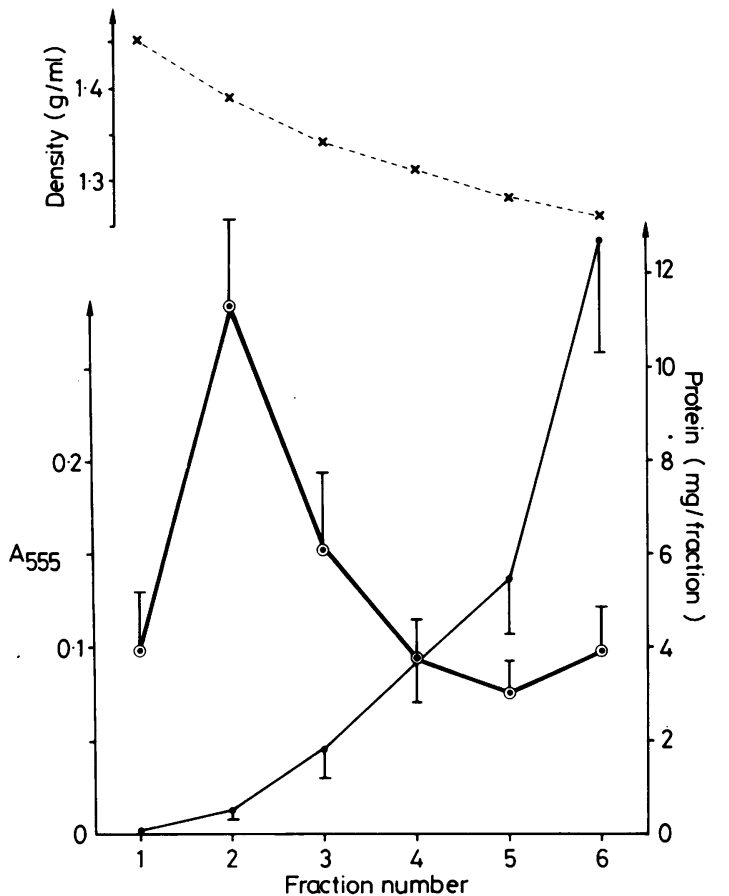

Fig. 2 Density gradient separation of aspirated rat gastric mucus glycoprotein. Open circles - PAS carbohydrate assay $\left(A_{555}\right)$. Dots-protein. Crosses-density. Error bars are ISD.

Consequently, the two densest fractions were always pooled and run on a Sepharose $2 \mathrm{BCL}$ column. This yielded a void volume peak (high molecular weight, HMW), and a broad partially included peak (low molecular weight, LMW, Fig. 3). Mercaptoethanol reduction of the crude aspirated mucus, followed by gradient density centrifugation, yielded

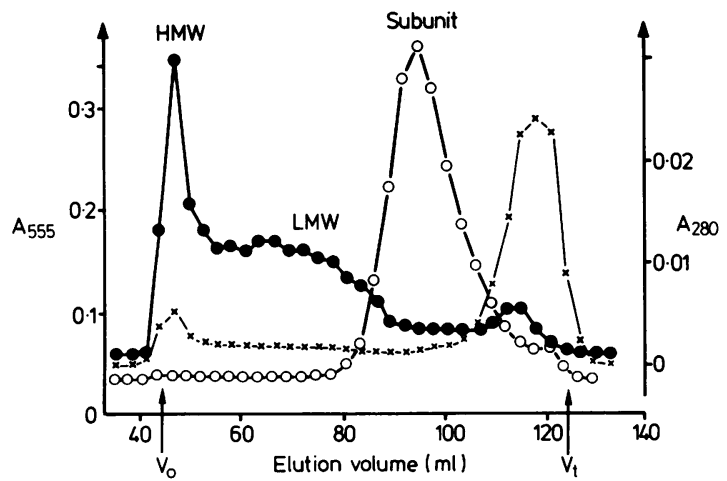

Fig. 3 Sepharose $2 B C L$ gel filtration profiles of pooled fractions 1 and 2 (Fig. 2). : absorbance of PAS carbohydrate assay $\left(A_{555}\right)$. $\bigcirc$ : after mercaptoethanol reduction $\left(A_{555}\right)$. $\times$ : absorbance at $280 \mathrm{~nm}\left(A_{280}\right)$. $V_{o}-$ void volume. $V_{1}$-total volume.
Table 2 Composition of $H M W$ and $L M W$ rat gastric mucus*

\begin{tabular}{|c|c|c|}
\hline & $\begin{array}{l}H M W \\
(\%)\end{array}$ & $\begin{array}{l}L M W \\
(\%)\end{array}$ \\
\hline Fucose $\dagger$ & $15 \cdot 6$ & $16 \cdot 5$ \\
\hline Galactose & $38 \cdot 2$ & $36 \cdot 8$ \\
\hline Mannose & $0 \cdot 6$ & $0 \cdot 3$ \\
\hline Glucose & $0 \cdot 8$ & 1.5 \\
\hline Glucuronic acid & $1 \cdot 1$ & $0 \cdot 6$ \\
\hline $\mathrm{N}$-acetyl galactosamine & $12 \cdot 8$ & $14 \cdot 7$ \\
\hline $\mathrm{N}$-acetyl glucosamine & $29 \cdot 8$ & $28 \cdot 6$ \\
\hline $\mathrm{N}$-acctyl neuraminic acid & $1 \cdot 3$ & 0.9 \\
\hline Aspartic acid $\ddagger$ & 0.46 & 0.52 \\
\hline Threonine & $(1 \cdot 0)$ & $(1 \cdot 0)$ \\
\hline Serine & $0 \cdot 88$ & 0.84 \\
\hline Glutamic acid & $0 \cdot 66$ & $0 \cdot 66$ \\
\hline Proline & 0.67 & 0.53 \\
\hline Glycine & 1.69 & 0.57 \\
\hline Alanine & 0.55 & 0.33 \\
\hline Valine & $0 \cdot 39$ & $0 \cdot 37$ \\
\hline
\end{tabular}

${ }^{*}$ Rat gastric mucus was aspirated from the mucosal surface, and separated on a $\mathrm{CsCl}$ density gradient, as described in Methods. The bottom $1.7 \mathrm{ml}$ of each density gradient tube was applied to a Sepharose 2BCL column, which resolved the mucus glycoprotcin into high molecular weight (HMW) and low molecular wcight (LMW) fractions (Fig. 3); † Sugars are expressed in mole per cent; $\ddagger$ Amino acids are expressed in mole per mole thrconine. Data shown for major amino acids ( $>0.3 \mathrm{~mol} / \mathrm{mol}$ thrconine $)$.

a single well included peak (Subunit, Fig. 3). These gel filtration profiles are similar to those obtained with human gastric mucus. ${ }^{2}$

The sugar compositions of the high molecular weight and low molecular weight fractions were similar (Table 2), and were typical of rat gastric mucus glycoprotein. ${ }^{6}$ The amino acid compositions were also typical of mucus glycoproteins, but the high molecular weight fraction had a higher, though variable, glycine content than the low molecular weight fraction. As expected, the sugar content was greater than the protein content. The sugar/amino acid weight ratio was 3.0 for high molecular weight, and 4.0 for low molecular weight. Mannose, glucose and glucuronic acid were each less than $2 \%$ of the total sugars. Consequently $\mathrm{N}$-linked glycoproteins, starch, and proteoglycans were not significant contaminants.

The minor peak at $115 \mathrm{ml}$ in the unreduced profile of Figure 3 was not included in the calculation of per cent high molecular weight. Analysis of the post-100 $\mathrm{ml}$ material gave $14 \mathrm{~mol} \%$ glucose and $3 \mathrm{~mol} \%$ mannose, suggesting the presence of significant quantities of non-mucus glycoprotein carbohydrate. In addition, the post $-100 \mathrm{ml}$ fractions had a high absorbance at $280 \mathrm{~nm}$, again indicating the presence of non-mucus glycoprotein material (Fig. 3). The 280 $\mathrm{nm}$ void volume peak was due to nucleic acids as it was abolished by treatment with DNAase and RNAase. Nuclease treatment, however, was not 
done routinely as the calculated nucleic acid content was not sufficient to cause significant interference in the PAS assay of the high molecular weight fractions. ${ }^{+}$

Gel filtration profiles of the combined two highest density fractions of mucus samples aspirated from 23 rats have been obtained. Considerable variation was noted in the percentage of the glycoprotein present in the high molecular weight form (mean \% HMW= $58.9 \% ; \mathrm{SD}=9 \cdot 6 \% ; \mathrm{n}=23)$. Assay variability $(\mathrm{SD}=$ $2.9 \% ; n=9$ ) accounted for only a small proportion of the observed variation.

\section{ASPIRIN DOSING AND ASSESSMENT OF MUCOSAL DAMAGE}

Eight rats (four male; four female) of 41 died during the aspirin dosing period. The cause of death was upper gastrointestinal bleeding in every case. In the 33 survivors, the six month aspirin regimen resulted in some animals having extensive gastric ulceration, while others had little or none. All chronic ulcers were confined to the glandular mucosa, and the majority were located close to the corpus-antrum junction.

Seventy nine chronic ulcers were identified by the stereoscopic criteria in this group (Table 1). The mean ulcer score was $4.4(\mathrm{SD}=3.5)$ and there was no significant difference between male and female rats $(p>0 \cdot 10)$. Of the 33 surviving aspirin dosed rats, 18 (eight male; 10 female) had had gastric mucus sampled by aspiration, before dosing, and 15 (six male; nine female) did not. There was no significant difference between the mean ulcer scores of these two groups $(p>0 \cdot 10)$, indicating that previous mucus sampling had not affected the amount of chronic mucosal damage induced by aspirin. Ten rats were dosed daily, for six months, with $1 \%$ methylcellulose only. Five males and four females survived. Four (one male; three female) had gastric mucus sampled by aspiration before dosing. Although a few erosions were present, there was only one ulcer, in the stomach of a mucus aspirated rat (ulcer score $=1$ ). Therefore, the dosing process, itself, had contributed very little to the mucosal damage produced by the chronic aspirin administration.

Three additional observers assessed the stomachs of 19 (randomly chosen) of the 33 aspirin dosed rats and gave ulcer scores which correlated closely with those reported here $(r=0 \cdot 87,0 \cdot 88,0 \cdot 57 ; p \leqslant 0.01)$.

Histological sections were taken through as many of the stereoscopically identified chronic ulcers as practicable. The stereoscopic diagnosis was confirmed in 20 of 22 cases. Sections were taken through 24 lesions which were stereoscopically assessed to be erosions and the diagnosis was confirmed in every case. The stereoscopic method of assessment was thus able to accurately identify chronic ulcers, and it could reliably discriminate between ulcers and erosions.

CORRELATION OF PER CENT HIGH MOLECULAR WEIGHT AND ULCER SCORE

Eighteen rats had gastric mucus sampled and analysed by gel filtration before dosing for six months with aspirin (eight males and 10 females). They were killed, and gastric ulceration was assessed. A significant negative correlation was found between per cent high molecular weight and ulcer scores for individual rats $(r=-0.85, p<0.001 ;$ Fig. 4$)$. An equally significant negative correlation was found between per cent high molecular weight and the number of chronic ulcers $(r=-0 \cdot 79, p<0 \cdot 001)$. Thus, gel filtration assay of mucus sampled from rats, before dosing with aspirin, had predictive value for the degree of subsequent chronic gastric ulceration.

\section{Discussion}

A number of mucosal defence factors are thought to protect the stomach from damage by acid and pepsin. These include the mucus-bicarbonate barrier, and cellular and vascular factors. ${ }^{\circ}$ The gastric mucus provides an 'unstirred layer' on the mucosa, into which bicarbonate ions are actively secreted. ${ }^{\prime}$ The result is that, at the apical surface of the gastric epithelial cells, the $\mathrm{pH}$ ranges from $6-7 \cdot 5$, and mucosal damage from acid and pepsin is opposed.

The mucus layer contains a polydisperse mixture of mucus glycoproteins which are responsible for its

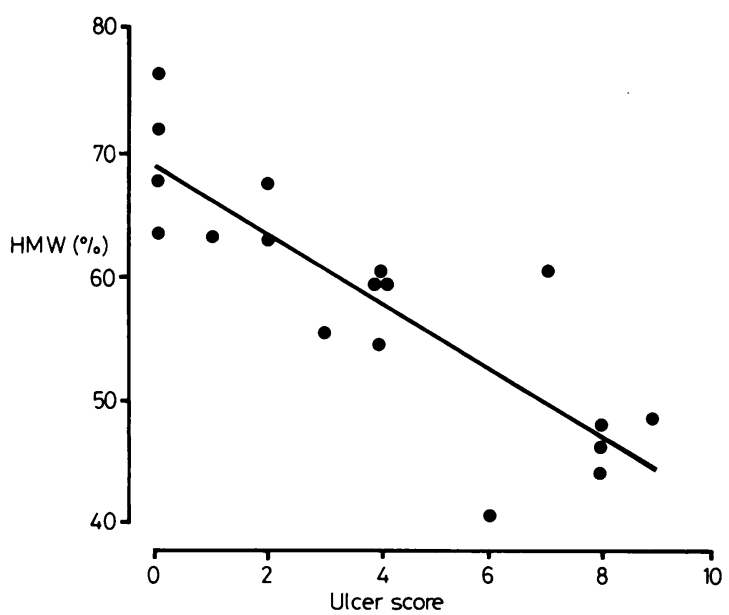

Fig. 4 Relationship between percentage of mucus glycoprotein in $\mathrm{HMW}$ form $(\% \mathrm{HMW})$, and degree of subsequent aspirin-induced chronic gastric ulceration (ulcer score). $r=-0 \cdot 85, n=18$. The line drawn is the regression line, with ulcer score as the independent variable. 
gel forming properties. Mercaptoethanol reduction, or extensive proteolysis, converts the glycoproteins to the subunit form, with the complete loss of these properties."' The weakening effect of lower molecular weight glycoproteins on the strength of the mucus gel, has already been discussed.' It has been previously suggested that the high molecular weight mucus glycoprotein at the luminal surface of the mucus layer is broken down by pepsin to the low molecular weight form, and that it is ultimately lost into the gastric lumen. The mucus layer is thus maintained by a balance between secretion by the surface epithelial cells, and degradation by the gastric juices. ${ }^{2}$ If mucus glycoprotein is secreted as the high molecular weight form, then the proportion of low molecular weight mucus glycoprotein found in a sample of gastric mucus is determined by the balance between the rate of mucus glycoprotein secretion, and the rate of peptic digestion. In the latter, variations between individuals would thus be owing either to differences in peptic activity, or to differences in the susceptibility of mucus glycoprotein to digestion. Alternatively, the high molecular weight and low molecular weight forms may be secreted in variable proportions. Whichever of these is the case, an assay which measures the proportion of mucus glycoprotein in the high molecular weight form may be expected to give an indication of the defensive value that a particular mucus layer has for its underlying mucosa. This scenario is consistent with our finding of a correlation between per cent high molecular weight and the degree of subsequent chronic gastric ulceration.

Kramer's group showed that the subunit structure, and the amino acid and carbohydrate composition of rat gastric mucus glycoprotein are similar to those in man and pig." For two reasons, however, this group did not observe the high molecular weight and low molecular weight forms which we have described. First, they studied water soluble mucus, which we have found to be mostly low molecular weight glycoprotein. They solubilised the water insoluble mucus (which we have found to contain the high molecular weight form) with mercaptoethanol, which converts it to the subunit form. Second, their gel filtration was carried out on Sepharose $4 B$, which does not separate the high molecular weight and low molecular weight forms.

Gel filtration separates according to molecular size, rather than by molecular weight. The nomenclature of other workers, ${ }^{2}$ however, using the same technique, has been used. Native mucus glycoprotein is polydisperse, but usually most of it elutes in a void volume fraction on Sepharose $2 \mathrm{BCL}$, which corresponds to our high molecular weight fraction. The average molecular weight of native mucus glyco- protein has been reported to be approximately $2 \times 10^{\prime \prime}$ for man, ${ }^{1}$ and pig. ${ }^{2}$ Also, the average molecular weight of the subunit form has been reported to be approximately $5 \times 10^{4}$ for pig. "2 The low molecular weight fraction is likely to be a polydisperse mixture with an average molecular weight intermediate between high molecular weight and subunit.

One of the current difficulties in the study of mucus glycoproteins is the lack of a widely accepted and specific assay method. We combined two commonly used purification techniques $(\mathrm{CsCl}$ density gradient centrifugation, and gel filtration) with a moderately sensitive, but non-specific, carbohydrate assay. The amino acid and sugar analyses confirm that the PAS carbohydrate assay was indeed measuring mainly mucus glycoprotein. Except for glycine, the sugar and amino acid analyses of both the high molecular weight and low molecular weight fractions were essentially the same. Whether the high glycine is an integral component of high molecular weight mucus glycoprotein, or merely a coeluting impurity, has yet to be determined. Differences between these two forms of mucus glycoprotein are currently being investigated.

Many animal models have been described for chronic gastric ulcer. ${ }^{1}$ The chronic aspirin dosed rat was chosen because it produces a wide range of responses. Also, some characterisation of rat mucus glycoprotein has been done. ${ }^{.13}$ Most important of all, the method is reproducible and produces chronic ulcers which, histologically, are very similar to their human counterpart. When compared with aspirin dosages in man, that used here appears high on a weight basis. Others, however, have found that similar dosages, in the rat, produce blood levels which correspond with the therapeutic range in man. ${ }^{3}$

The majority of the effects of aspirin which have been studied are acute, and relatively little is known about the chronic effects. ${ }^{14}$ 15 Two important differences in these effects are the type and distribution of the lesions produced. Acute erosions, which do not penetrate the mucularis mucosae, are produced within an hour of aspirin administration. ${ }^{16}{ }^{17}$ Chronic gastric ulcers are only seen after repeated administration for six months. ${ }^{3}$ The distribution of the lesions also differs. Erosions are scattered over the mucosa of the corpus and antrum, ${ }^{1+16}$ whereas, chronic ulcers are usually confined to the antrum and corpusantrum junction. ${ }^{3}$ In addition, acute erosions heal more rapidly ${ }^{1617}$ than chronic ulcers. ${ }^{3}$

A possible explanation for the differences between individual rats in the degree of chronic gastric ulceration is that aspirin may produce a depression in one or more of the mucosal defence factors, and thus reveal an underlying variability in the mucus layer. For example, as discussed earlier, in susceptible animals, 
a weaker mucus gel structure may be because of smaller proportions of high molecular weight glycoprotein. Such a mechanism is supported by our findings that the per cent high molecular weight mucus glycoprotein is inversely correlated with the degree of chronic gastric ulceration. This variability in mucus is present normally in the rat; it is not caused by the aspirin, as this was given after the mucus samples were taken. This is the first time that such a pre-existent mucosal susceptibility has been demonstrated.

There are obviously many dangers in extrapolation from animal models to man. The comparison with the observation that patients with chronic gastric ulcers have a lower proportion of high molecular weight mucus glycoprotein in their gastric mucus layer is striking." The results obtained with the rat model suggest that this variability may also predate chronic gastric ulceration in man. Consequently, an assay of per cent high molecular weight mucus glycoprotein may be useful in identifying those likely to develop chronic gastric ulceration. A relatively non-invasive method must be found, for the measurement of gastric mucus quality in man, in order that epidemiological studies can be undertaken.

This investigation was supported by the Medical Research Council of New Zealand, and the Canterbury Medical Research Foundation, New Zealand.

\section{References}

1 Pfeiffer CJ, MacPherson BR. Experimental Gastric Ulcer. In: Mozsik GY, Javor T, eds. Progress in peptic ulcer. Budapest: Akademiai Kiado, 1976: 353-79.

2 Younan F, Pearson J, Allen A, Venables C. Changes in the structure of mucous gel on the mucosal surface of the siomach in association with peptic ulcer disease. Gastroenterology 1982; 82: 827-31.

3 St John DJB, Yeomans ND, De Boer WGRM. Chronic gastric ulcer induced by aspirin: an experimental model. Gastroenterology 1973; 65: 634-41.

4 Mantle M, Allen A. A colorimetric assay for glycoprotein based on the Periodic Acid/Schiff stain. Biochem Soc Trans 1978; 6: 607-9.
5 Lowry OH, Rosebrough NJ, Farr AL, Randall RJ. Protein measurement with the Folin phenol reagent. J Biol Chem 1951; 193: 265-75.

6 Spee-Brand R, Strous Ger JAM, Kramer MF. Isolation and partion characterisation of rat gastric mucus glycoprotein. Biochim Biophys Acta 1980; 621: 104-16.

7 Zanetta JP, Breckenridge WC, Vincendon G. Analysis of monosaccharides by gas-liquid chromatography of the O-methyl glycosides as trifluoro-acetate derivatives. J Chromatogr 1972; 69: 291-304.

8 O'Brien PE. The nature of the mucosal defences against ulceration. In: Carter DC, ed. Peptic ulcer. Edinburgh: Churchill Livingstone, 1983: 28-43.

9 Williams SE, Turnberg LA. Demonstration of a $\mathrm{pH}$ gradient across mucus adherent to rabbit gastric mucosa: evidence for a mucus-bicarbonate barrier. Gut 1981; 22: 94-6.

10 Bell AE, Sellers LA, Allen A, Cunliffe WJ, Morris ER, Ross-Murphy SB. Properties of gastric and duodenal mucus: Effect of proteolysis, disulfide reduction, bile, acid, ethanol, and hypertonicity on mucus gel structure. Gastroenterology 1985; 88: 269-80.

11 Pearson J, Allen A, Venables C. Gastric mucus: isolation and polymeric structure of the undegraded glycoprotein: Its breakdown by pepsin. Gastroenterology 1980; 78: 709-15.

12 Starkey BJ, Snary D, Allen A. Characterization of gastric mucoproteins isolated by equilibrium densitygradient centrifugation in caesium chloride. Biochem $J$ 1974; 141: 633-9.

13 Ohara S, Ishihara K, Kakei M, Azuumi Y, Hotta K. Distribution of mucosal macromolecular glycoproteins in rat stomach. Comp Biochem Physiol 1982; 72B: $309-11$.

14 St John DJB, Yeomans ND, McDermott FT, De Boer WGRM. Adaption of the gastric mucosa to repeated administration of aspirin in the rat. Am J Dig Dis 1973; 18: 881-6.

15 Eastwood GL, Quimby GF. Effect of chronic aspirin ingestion on epithelial proliferation in rat fundus, antrum, and duodenum. Gastroenterology 1982; 82: 852-6.

16 Azuumi Y, Ohara S, Ishihara K, Okabe H, Hotta K. Correlation of quantitative changes in mucosal glycoproteins with aspirin-induced gastric damage in rats. $G u t$ 1980; 21 : 533-6.

17 Yeomans ND, St John DJB, De Boer WGRM. Regeneration of gastric mucosa after aspirin-induced injury in the rat. Am J Dig Dis 1973; 18: 773-80. 